\section{CD6 targeting}

CD6 is a highly specific marker of T cells and a risk factor for multiple sclerosis; however, its cellular function in the latter context is unclear. In the Proceedings of the National Academy of Sciences, Lin and colleagues develop a CD6-knockout mouse (KO) and a mouse with knock-in human CD6 on a CD6-knockout background $(\mathrm{KI})$ to investigate $\mathrm{CD} 6$ 's role in experimental autoimmune encephalitis. The $\mathrm{KO}$ mice are almost totally resistant to this disease and show less induction of the $T_{H} 1$ and $T_{H} 17$ subsets of helper T cells in vitro. Although KO T cells have higher expression of activation markers, which suggests that CD6 is a negative regulator, they show less proliferation, a greater propensity to die by apoptosis and impaired transmigration across endothelial layers. Finally, antibodies generated against human CD6 protect $\mathrm{KI}$ mice in a non-depleting manner. CD6 is probably a negative regulator of the activation of $\mathrm{T}$ cells and also seems to be required for their survival and entry into the central nervous system and could be an important target in multiple sclerosis.

Proc. Natl. Acad. Sci. USA (16 February 2017) doi:10.1073/ pnas. 1615253114

\section{Maintaining $\mathrm{T}_{\mathrm{RM}}$ cells}

Tissue-resident memory $\mathrm{T}$ cells ( $\mathrm{T}_{\mathrm{RM}}$ cells) persist long term at epithelial barrier surfaces and are essential in anti-pathogen responses at these sites. In Nature, Kupper and colleagues demonstrate that mouse $\mathrm{T}_{\mathrm{RM}}$ cells in the skin are characterized by high expression of the fatty-acid-binding proteins FABP4 and FABP5. This expression is critical for the maintenance

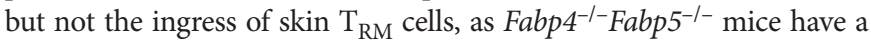
much lower abundance of these cells and are vulnerable to experimental infection with vaccinia virus. Consistent with their high expression of FABP4 and FABP5, skin $\mathrm{T}_{\mathrm{RM}}$ cells show enhanced uptake and metabolism of free fatty acids. Finally, human psoriatic lesions also contain $\mathrm{T}_{\mathrm{RM}}$ cells that express FABP4 and FABP5. Not only do these data reveal an essential and cell-type-specific mechanism for the maintenance of $\mathrm{T}_{\mathrm{RM}}$ cells but they also offer a possible means of targeting this population.

Nature (20 February 2017) doi:10.1038/nature21379

\section{Antimicrobial IL-22}

Interleukin 22 (IL-22) protects hosts against microbial infection at barrier tissues. In Science Immmunology, Sakamoto et al. show that IL-22 also induces host resistance to systemic infection by pathogenic gut bacteria. Intravenous infection with Citrobacter rodentium or Escherichia coli induces IL-22 that induces liver production of acute-phase response proteins, including the heme scavenger hemopexin (HPX). $1122^{-1-}$ or $\mathrm{Hpx}^{I_{-}}$mice are unable to control bacterial proliferation in blood and succumb to infection by pathogens that express hemolysins or other virulence proteins that liberate host heme complexes. Administration of HPX rescues $1122^{-1-}$ mice from death after bacterial blood infection, which suggests that sequestration of heme is sufficient to retard bacterial growth until other immune responses develop to control the infection.

Sci. Immunol. 2, eaai8371 (2017)

Written by Laurie A. Dempsey, Zoltan Fehervari \& Ioana Visan

\section{Atherosclerosis-prone HSCs}

Age-related accumulation of mutations in the gene encoding the methylcytosine dioxygenase TET2 in hematopoietic cells is associated with a greater incidence of vascular pathology in humans. In Science, Walsh and colleagues show that reconstitution of atherosclerosis-prone Ldlr ${ }^{-1-}$ mice with Tet2 ${ }^{-/-}$hematopoietic stem cells (HSCs) increases the size of atherosclerotic plaques. Tet2 ${ }^{-/-}$HSCs clonally expand in the immune-cell pool of recipient mice with no effect on blood cell counts, body weight, systemic insulin, glucose or cholesterol, or the proliferation or apoptosis of macrophages in the plaque. Myeloid-cellrestricted deletion of Tet 2 has similar effects. In vitro, Tet $2^{-/-}$macrophages exposed to low doses of oxidized low-density lipoprotein and the cytokines TNF and IFN- $\gamma$ to mimic the conditions in atherosclerotic plaques have higher expression of IL-1 $\beta$ due to increased expression of pro-IL-1 $\beta$ and increased activity of the NLRP3 inflammasome. In vivo, Tet $2^{-1-}$ monocytes undergo more recruitment to the aortic wall than do wild-type cells. The NLRP3 inhibitor MCC950 diminishes plaque size to that in $\mathrm{Ldlr}^{-/-}$mice. These observations link age-related somatic mutations in HSCs with atherosclerosis.

Science 355, 842-847 (2017)

\section{Importing material}

Conversion of the non-essential amino acid serine into glycine as part of one-carbon metabolism is a key metabolic node in proliferating cells. Serine can be synthesized from glucose or can be taken up from exogenous sources. In Cell Metabolism, Jones and colleagues report that extracellular serine is critical for the proliferation of T cells after antigen stimulation. Genes encoding products involved in serine biosynthesis are upregulated in activated $C D 8^{+} \mathrm{T}$ cells in humans and mice, but $75 \%$ of the serine pool is derived from extracellular sources. Mice on a serineand glycine-restricted diet show less population expansion of antigen-specific $\mathrm{CD}^{+}$and $\mathrm{CD} 4^{+} \mathrm{T}$ cells and diminished secondary responses, while $\mathrm{T}$ cells cultured in serine-free medium show less proliferation but normal expression of activation markers, cytokine secretion and glucose bioenergetics. The conversion of extracellular serine to glycine is essential for nucleotide synthesis in proliferating $\mathrm{T}$ cells.

Cell Metab. 25, 345-357 (2017)

\section{Adipose-tissue ILCs}

Multiple types of immune cells are found in adipose tissues. In Immunity, Boulenouar et al. characterize type 1 innate lymphoid cells (ILCs) that reside in adipose tissues in both lean people and obese people. Adiposetissue ILCs are a heterogeneous population that dynamically changes in response to high-fat diets and includes cytotoxic natural killer cells. Adipose tissue ILCs are phenotypically different from similar populations in spleen and liver. Curiously, in lean people, adipose-tissue ILCs can kill tissue-resident M2-polarized macrophages, which express the stress ligand RaeI recognized by natural killer cells. Adipose-tissue ILCs from obese people have diminished cytolytic activity; however, adoptive transfer of ILCs from lean donors enhances glucose intolerance in obese recipients. These findings suggest that ILCs contribute to the homeostasis of adipose-tissue macrophages, although fuller understanding of their interactions will require further study.

$L A D$

Immunity 46, 273-286 (2017) 